\title{
Combinational effects of prebiotic oligosaccharides on bifidobacterial growth and host gene expression in a simplified mixed culture model and neonatal mice
}

\author{
Tatsuya Ehara*, Hirohisa Izumi, Muneya Tsuda, Yuki Nakazato, Hiroshi Iwamoto, Kazuyoshi Namba \\ and Yasuhiro Takeda \\ Nutritional Science Institute, Morinaga Milk Industry Co. Ltd, 5-1-83, Higashihara, Zama, Kanagawa 252-8583, Japan
}

(Submitted 24 November 2015 - Final revision received 3 March 2016 - Accepted 18 April 2016)

\section{Abstract}

It is important to provide formula-fed infants with a bifidobacteria-enriched gut microbiota similar to those of breastfed infants to ensure intestinal health. Prebiotics, such as certain oligosaccharides, are a useful solution to this problem, but the combinational benefits of these oligosaccharides have not been evaluated. This study investigated the benefits of oligosaccharide combinations and screened for an optimal combination of oligosaccharides to promote healthy gut microbiota of formula-fed infants. In vitro and in vivo experiments were performed to assess the bifidogenic effects of lactulose (LAC) alone and LAC combined with raffinose (RAF) and/or galacto-oligosaccharide (GOS), using a mixed culture model and neonatal mice orally administered with these oligosaccharides and Bifidobacterium breve. In the in vitro culture model, the combination of the three oligosaccharides (LAC-RAF-GOS) significantly increased cell numbers of B. breve and Bifidobacterium longum $(P<0.05)$ compared with either LAC alone or the combination of two oligosaccharides, and resulted in the production of SCFA under anaerobic conditions. In the in vivo experiment, the LAC-RAF-GOS combination significantly increased cell numbers of $B$. breve and Bacteroidetes in the large intestinal content $(P<0.05)$ and increased acetate concentrations in the caecal content and serum of neonatal mice. Genes related to metabolism and immune responses were differentially expressed in the liver and large intestine of mice administered with LAC-RAF-GOS. These results indicate a synergistic effect of the LAC-RAF-GOS combination on the growth of bifidobacteria and reveal possible benefits of this combination to the gut microbiota and health of infants.

\section{Key words: Oligosaccharides: Bifidobacteria: Infant formula: Gut microbiota}

Gut microbiota of breastfed infants are dominated by bifidobacteria $^{(1,2)}$. Bifidobacteria contribute to infant health by preventing certain conditions, such as infections ${ }^{(3-5)}$ and allergies $^{(6,7)}$. The physiological functions of SCFA, such as acetate, produced by bifidobacteria and the direct modulation of the host immune response by bifidobacteria may underlie these preventive effects ${ }^{(3,8,9)}$. Bifidobacteria populations are often smaller in the gut microbiota of formula-fed infants than in breastfed infants ${ }^{(1,2)}$. Breastfed infants also have decreased infection risks and fewer allergies than formula-fed infants ${ }^{(10-14)}$. Thus, exclusive breastfeeding is strongly recommended for optimal development of gut microbiota, defence molecules and growth factors $^{(15,16)}$. Therefore, an important challenge for the development of infant formula is to provide infants who cannot be breastfed sufficiently with a bifidobacteria-enriched gut microbiota similar to that of breastfed infants.

Non-digestive oligosaccharides, a class of prebiotics, can selectively promote the growth of bifidobacteria by providing an energy source specific for bifidobacteria ${ }^{(17-19)}$. More than
200 types of structurally distinct oligosaccharides have been found in human breast milk ${ }^{(18,20)}$. These human milk oligosaccharides (HMO) are thought to contribute to the development of the bifidobacteria-dominated gut microbiota in breastfed infants ${ }^{(21)}$. The structural diversity of HMO appears to be important to the superior bifidogenic properties of human milk, as oligosaccharides with different structural features require different microbial metabolic processes to be utilised and therefore exhibit different prebiotic properties ${ }^{(19,22)}$. Infant formula has traditionally relied on alternative commercially available non-digestive oligosaccharides for their bifidogenic effects, as the diverse and complex HMO are difficult to industrially reproduce. Indeed, there is evidence of prebiotic effects in infant formulas containing commercially available non-digestive oligosaccharides, such as lactulose (LAC) ${ }^{(23,24)}$, raffinose $(\mathrm{RAF})^{(25)}$, galacto-oligosaccharide $(\mathrm{GOS})^{(26)}$ and fructo-oligosaccharide ${ }^{(27)}$. However, the microbiota composition of infants fed these formulas is different from that of breastfed infants ${ }^{(28)}$. Therefore, it is important to clarify and

Abbreviations: GOS, galacto-oligosaccharide; JCM, Japan Collection of Microorganisms; LAC, lactulose; RAF, raffinose; Th, helper T cell.

* Corresponding author: T. Ehara, fax +81 46252 3055, email t-ehara@morinagamilk.co.jp 
validate the bifidogenic effects of these oligosaccharides on the infant gut microbiota. In vitro fermentation systems using infant faeces have been developed ${ }^{(29,30)}$ to screen for better oligosaccharides, but studies using human faecal samples are complicated and less reproducible.

Because different oligosaccharides exhibit different prebiotic properties $^{(19)}$, we hypothesised that a combination of oligosaccharides would promote bifidobacteria growth in the gut microbiota of infants better than each oligosaccharide alone. This study evaluated the effect of oligosaccharide combinations on the growth of Bifidobacterium breve and Bifidobacterium longum, two major bifidobacteria species found in the gut of breastfed infants ${ }^{(21)}$. We used an in vitro mixed culture model of artificial infant microbiota that was previously reported in a mouse model with humanised microbiota ${ }^{(31)}$. We also conducted an in vivo experiment using neonatal mice to validate the results of our in vitro experiment. We selected LAC as a basic oligosaccharide because it is one of the most time-proven prebiotics and has been used in commercial infant formula products for about 50 years ${ }^{(24)}$. We assessed the combination of LAC with RAF and/or GOS.

\section{Methods}

\section{Oligosaccharides}

LAC (MLC-97, 99.9\%; Morinaga Milk Industry Co. Ltd), RAF (Nitten Raffinose, >98\%; Nippon Beet Sugar Manufacturing Co. Ltd) and GOS (composed of approximately $14 \%$ tetrasaccharides, $82 \%$ trisaccharides and 2\% disaccharides) were used in this study. GOS was obtained by purification of Oligomate $55 \mathrm{~N}$ (Yakult Honsha Co. Ltd) using gel filtration column chromatography, as previously reported ${ }^{(29)}$.

\section{Bacterial strains}

Bacterial strains were obtained from the American Type Culture Collection (ATCC) and the Japan Collection of Microorganisms (JCM). Seven bacterial species were used to create the artificial human baby flora culture ${ }^{(31)}$ : $B$. breve $(\mathrm{M}-16 \mathrm{~V}$; Morinaga Milk Industry) ${ }^{(32)}$, B. longum $\left(\mathrm{JCM}^{2} 217^{\mathrm{T}}\right.$ ), Escherichia coli $\left(\mathrm{JCM} 1649^{\mathrm{T}}\right)$, Staphylococcus aureus (JCM20624 ${ }^{\mathrm{T}}$ ), Staphylococcus epidermidis (JCM2414 ${ }^{\mathrm{T}}$ ), Clostridium perfringens (ATCC13124 $4^{\mathrm{T}}$ ) and Parabacteroides distasonis (JCM5825 ${ }^{\mathrm{T}}$ ).

\section{In vitro fermentation experiment}

The seven bacterial strains were cultured individually at $37^{\circ} \mathrm{C}$ in Gifu Anaerobic Medium broth (Nissui Pharmaceutical Co. Ltd) to inoculate the test medium. Optical densities of cultures were monitored and bacteria were collected during growth phase by centrifugation. All strains were resuspended in a complex medium (FM medium $)^{(29,30)}$ containing yeast extract $(5 \mathrm{~g} / \mathrm{l})$, ascorbic acid $(10 \mathrm{~g} / \mathrm{l})$, sodium acetate $(10 \mathrm{~g} / \mathrm{l}),\left(\mathrm{NH}_{4}\right)_{2} \mathrm{SO}_{4}$ $(5 \mathrm{~g} / \mathrm{l})$, urea $(2 \mathrm{~g} / \mathrm{l}), \mathrm{MgSO}_{4} \cdot 7 \mathrm{H}_{2} \mathrm{O}(0 \cdot 2 \mathrm{~g} / \mathrm{l}), \mathrm{FeSO}_{4} \cdot \mathrm{H}_{2} \mathrm{O}(0 \cdot 01 \mathrm{~g} / \mathrm{l})$, $\mathrm{MnSO}_{4} \cdot \mathrm{H}_{2} \mathrm{O}(0 \cdot 007 \mathrm{~g} / \mathrm{l}), \mathrm{NaCl}(0 \cdot 01 \mathrm{~g} / \mathrm{l})$, Tween 80 ( $\left.1 \mathrm{~g} / \mathrm{l}\right)$, hemin $(0.05 \mathrm{~g} / \mathrm{l})$ and L-cysteine hydrochloride monohydrate $(0.5 \mathrm{~g} / \mathrm{l})$, and $2 \times 10^{7}$ cells $/ \mathrm{ml}$ of each strain were then pooled together.
This bacterial mixture was used as a seed culture. The in vitro fermentation was performed according to a previous report ${ }^{(29)}$ using a pH-controlled, multi-channel fermenter (Bio Jr.8; ABLE). In brief, the test medium was prepared as follows: $100 \mathrm{ml}$ of FM medium containing either LAC, LAC-RAF, LAC-GOS or LAC-RAF-GOS (total oligosaccharide concentration of $1 \% \mathrm{w} / \mathrm{v}$ ) was incubated with $\mathrm{CO}_{2}$ gas overnight to achieve anaerobic conditions at a $\mathrm{pH}$ of $7 \cdot 0$. The ratios of oligosaccharides in the culture medium were 1:1 and 1:1:1 for two- and three-oligosaccharide combinations, respectively. The test medium was inoculated with $1 \mathrm{ml}$ of the seed culture to create the artificial human baby flora, resulting in test medium containing $2 \times 10^{5}$ cells of each strain. After inoculation, fermentation was carried out at $37^{\circ} \mathrm{C}$ with continuous $\mathrm{CO}_{2}$ flow, gentle stirring and no $\mathrm{pH}$ regulation. After a $24-\mathrm{h}$ fermentation, $1 \mathrm{ml}$ of the test culture was sampled to measure bacterial cell numbers and SCFA.

\section{Measurement of bacterial cell numbers}

Bacterial cell numbers in the culture and in the large intestine content of mice were determined by a real-time PCR method using primers specific to the $16 \mathrm{~S}$ rRNA gene of each bacterial group, as previously reported ${ }^{(29)}$. Bacterial pellets from $1 \mathrm{ml}$ of the mixed culture, or approximately $20 \mathrm{mg}$ of the large intestinal content from neonatal mice, were vigorously homogenised in $500 \mu \mathrm{l}$ of extraction buffer ( $100 \mathrm{~mm}$-Tris-HCl, $40 \mathrm{~mm}$-EDTA and $1 \%$ of SDS at $\mathrm{pH} 9 \cdot 0$ ) with $300 \mathrm{mg}$ of $0 \cdot 1-\mathrm{mm}$-diameter glass beads and $500 \mu \mathrm{l}$ of TE (Tris-EDTA; $10 \mathrm{mM}$-Tris- $\mathrm{HCl}$, 1 mM-EDTA, pH 8.0)-saturated phenol for $30 \mathrm{~s}$ using the FastPrep FP100A bead cell disruptor (Qbiogene Inc.) at a power level of 5.0. Bacterial DNA was extracted and purified using a standard phenol-chloroform method. Purified DNA was subjected to realtime PCR using the 7500 Fast Real-Time PCR System (Life Technologies) and SYBR Premix Ex Taq (Takara Bio Inc.) with primers specific to the $16 \mathrm{~S}$ rRNA gene of each bacterial group to determine the cell numbers of each bacterial strain in the samples. Standard DNA for each bacterial strain was also prepared from a pure culture of each strain, in which the bacterial cell number was microscopically counted. These standard DNA were subjected to real-time PCR together with samples to construct standard curves for the determination of bacterial cell numbers in the samples. Detailed primer information is summarised in the online Supplementary Table S1.

\section{Measurement of SCFA}

SCFA were measured as previously described ${ }^{(29)}$. In brief, culture supernatants were diluted 5-fold with $3.75 \mathrm{~mm}-$ perchloric acid and then ultrafiltrated using a NANOCEP $10 \mathrm{~K}$ Omega spin column (Pall Corporation). Caecum contents from neonatal mice were homogenised in ten volumes of distilled water and then centrifuged at $13000 \mathrm{~g}$ for $10 \mathrm{~min}$ at $4^{\circ} \mathrm{C}$. Nine volumes of the resulting supernatant were mixed with one volume of $30 \mathrm{~mm}$-perchloric acid and then ultrafiltrated as described above. The concentrations of SCFA in ultrafiltrated samples were analysed using an ion-exclusion HPLC system 
(Shimadzu) $^{(29)}$. Concentrations of acetate in the culture supernatants were determined by subtracting the initial acetate concentration of FM medium from the acetate concentrations after fermentation.

\section{Measurement of lactulose}

Culture supernatants were ultrafiltrated using a NANOCEP $10 \mathrm{~K}$ Omega spin column. Oligosaccharides in the ultrafiltrated samples were separated by an ion-exclusion HPLC with the following conditions: column, a TSKgel SUGAR AXI column $(4.6 \times 150 \mathrm{~mm})$ (Tosoh); column temperature, $60^{\circ} \mathrm{C}$; flow rate, $0.4 \mathrm{ml} / \mathrm{min}$; mobile phase, $0.5 \mathrm{~m}$-borate buffer, $\mathrm{pH} 8.7$. Then, separated LAC was derivatised with arginine and detected using the post-column fluorometric detection method ${ }^{(33)}$ with a RF-20Axs fluorescence detector (Shimadzu). Consumption rates of LAC in the culture supernatants were determined by subtracting the concentrations of LAC remaining after the 24-h fermentation from the initial concentrations.

\section{Animal studies}

All animal experiments were approved by and performed in accordance with the guidelines of the Animal Research Committee of Morinaga Milk Industry Co. Ltd. Lactating female C57BL6 mice aged 10 weeks old with 2-d-old (D2) male pups were obtained from Japan SLC Inc. The litter size was adjusted to four. Mothers were fed ad libitum with a standard rodent diet (F-2; Funabashi Farm) and pups were nursed by their mothers throughout the study. The mice were exposed to a $12 \mathrm{~h}$ light $-12 \mathrm{~h}$ dark cycle and the room was maintained at a constant temperature of 23 ( $\operatorname{sem~} 2)^{\circ} \mathrm{C}$. To evaluate the effects of the oligosaccharide combinations on the growth of bifidobacteria, pups were divided into five groups and orally administered with $100 \mu \mathrm{l}$ of PBS containing one of the following five mixtures once a day from D6 to D14: vehicle (PBS alone), $5 \times 10^{7} B$. breve, $20 \mathrm{mg}$ LAC with $5 \times 10^{7} B$. breve, $20 \mathrm{mg}$ LAC-RAF mixture with $5 \times 10^{7} \quad B$. breve and $20 \mathrm{mg}$ LAC-RAF-GOS mixture with $5 \times 10^{7} B$. breve. The $\mathrm{w} / \mathrm{v}$ ratio of oligosaccharides was 1:1 and 1:1:1 for the LAC-RAF and LACRAF-GOS groups, respectively. Pups were killed at D15 by deep anaesthesia with sevoflurane to harvest the large intestine and caecum contents. The detailed protocol of the experiment is illustrated in Fig. 2(A).

\section{Quantitative real-time PCR analysis}

Total RNA in the liver and large intestine were extracted using TRIzol reagent (Life Technologies). The quality of the purified total RNA was confirmed by electrophoretic analysis using an Experion Automated Electrophoresis System (Bio-Rad Laboratories Inc.). Complementary DNA was synthesised from $1 \mu \mathrm{g}$ of total RNA using the QuantiTect Reverse Transcription Kit (Qiagen). Gene expression levels were determined by realtime PCR using the 7500 Fast Real-Time PCR System (Life Technologies) with SYBR Premix Ex Taq (Takara Bio). The specificity of each PCR was confirmed by analysing the melting curve of the amplicon according to the manufacturer's protocols. All samples amplified by real-time PCR were tested in duplicate. Relative mRNA levels of target genes were determined on the basis of the mRNA levels of the housekeeping gene $\beta$-actin $(A c t b)$. Detailed primer information is summarised in the online Supplementary Table S2.

\section{Statistical analysis}

Data are expressed as means with their standard errors. Differences in bacterial cell numbers, $\mathrm{pH}$ of each time point and concentrations of SCFA among the four experimental groups in the in vitro experiment and differences in bacterial cell numbers, concentrations of SCFA and mRNA levels among the five experimental groups in the in vivo experiments were all assessed by the Tukey-Kramer honestly significant difference (HSD) test for multiple comparisons. Data were analysed using JMP software version 5.1 (SAS institute Inc.). All results with $P<0.05$ were considered statistically significant.

\section{Results}

In vitro bifidogenic effects of lactulose and oligosaccharide combinations

We analysed the bifidogenic effects of LAC alone and various combinations of oligosaccharides using a simplified in vitro mixed culture model of artificial infant microbiota. After a 24-h fermentation, the cell numbers of $B$. breve in the cultures with the LAC-GOS combination and with LAC-RAF-GOS combination were significantly higher than those in the cultures with LAC or LAC-RAF $(P<0.05)$ (Fig. 1(A)). The cell numbers of $B$. longum were significantly higher in the cultures with LACRAF-GOS compared with those in other tested cultures $(P<0.05)$ (Fig. 1(A)). Rapid decreases in $\mathrm{pH}$ were observed in the cultures with LAC-GOS and with LAC-RAF-GOS (Fig. 1(B)). Consistent with the cell number of bifidobacteria and the decrease in $\mathrm{pH}$ in the cultures after a 24-h fermentation, the concentrations of LAC were decreased by 19.1 (SEM 1.0) \% in the LAC culture and by 54.2 (SEM 6.2) \% in the LAC-RAF culture, respectively. In contrast, LAC was not detected in the LAC-GOS and LAC-RAF-GOS cultures after the 24-h fermentation. Consistent with this, concentrations of acetate, a major SCFA produced by the fermentation of carbohydrates by bifidobacteria, were significantly higher in the cultures with LAC-GOS and LAC-RAF-GOS than in the cultures with LAC and LAC-RAF $(P<0.05)$ (Fig. 1(C)), indicating active utilisation of the LAC-GOS and LAC-RAF-GOS combinations. The cell numbers of Staphylococcus were relatively high in the cultures containing LAC-RAF, the cell numbers of $E$. coli were high in LAC-GOS and LAC-RAF-GOS, the cell numbers of $C$. perfringens were high in LAC and the cell numbers of $P$. distasonis were high in LAC-RAF (Fig. 1(A)).

In vivo bifidogenic effects of lactulose, lactulose-raffinose and lactulose-raffinose-galacto-oligosaccharide

We conducted an in vivo experiment to validate the results of our in vitro culture model. We selected three oligosaccharide 


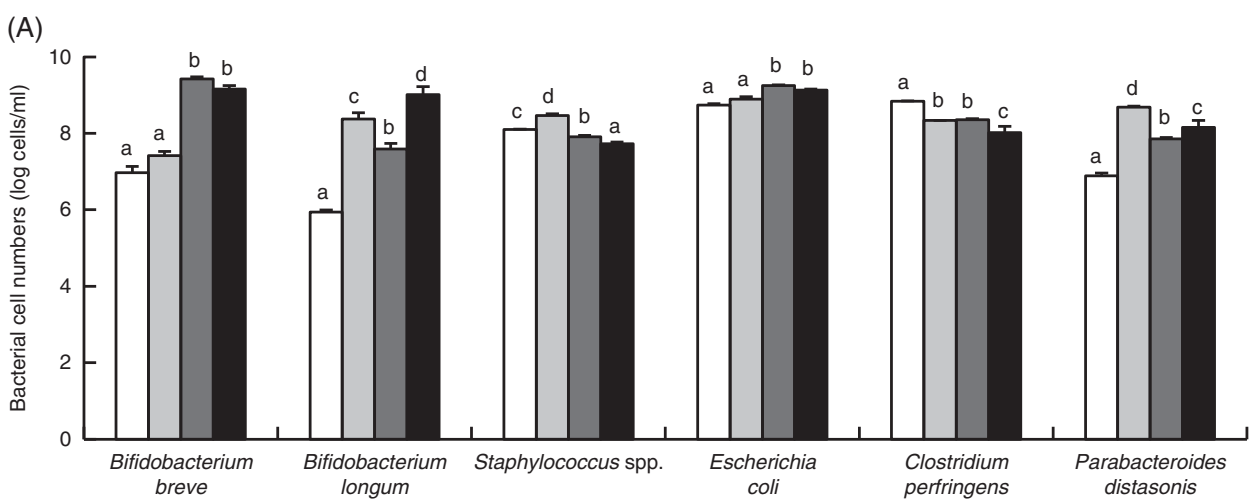

(B)

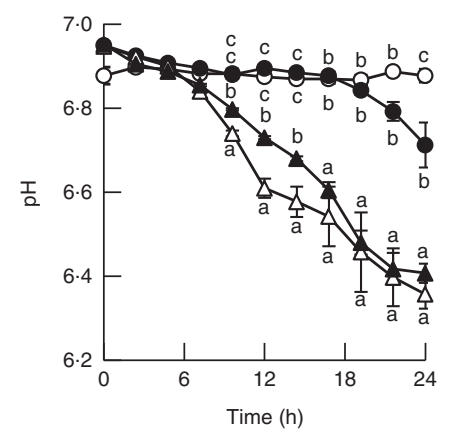

(C)

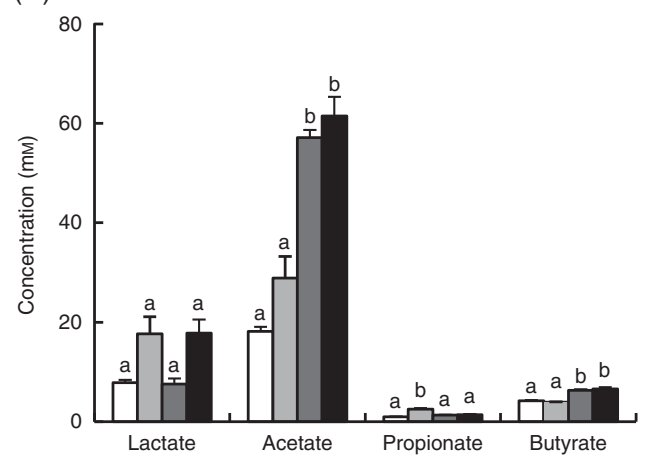

Fig. 1. Combinational effects of oligosaccharides on the bifidobacterial growth in vitro. (A) Bacterial cell numbers of each species in the culture after the 24-h fermentation. (B) $\mathrm{pH}$ changes of the culture medium during fermentation. (C) Concentrations of lactate and SCFA in the culture medium after the 24-h fermentation. Values are means $(n 4)$, with their standard errors. ${ }^{a, b, c, d}$ Mean values with unlike letters were significantly different $(P<0.05$, Tukey-Kramer HSD test). $\square$, lactulose (LAC); $\square$, LAC-raffinose (RAF); $\square$, LAC-galacto-oligosaccharide (GOS); $\square$, LAC-RAF-GOS; —O—, LAC; ——, LAC-RAF; $\square-$, LAC-GOS; $\longrightarrow$ - LAC-RAF-GOS.

groups, LAC, LAC-RAF and LAC-RAF-GOS, that appeared to differ in their bifidogenic effects according to the in vitro model for further in vivo analysis.

In the in vivo experiment, neonatal mice were orally administered with one of the oligosaccharide mixtures and $B$. breve from D6 to D14 (Fig. 2(A)). The cell numbers of $B$. breve in the large intestine content were measured after a 9-d administration by real-time PCR. 16S rRNA gene copy numbers of total bacteria were slightly higher in the $B$. breve-administered groups than in the vehicle group (Fig. 2(B)). B. breve were not detected in the vehicle group, but they were detected in all groups administered with $B$. breve at about $10^{10}$ cells/g of faeces (Fig. 2(B)). Levels of $B$. breve $16 \mathrm{~S}$ rRNA gene in the gut reflected the trend observed in the in vitro experiment depicted in Fig. 1. The levels of B. breve $16 \mathrm{~S}$ rRNA gene were relatively low in the LAC group (accounting for $0.8 \%$ of all bacterial 16S rRNA gene, a similar level to the $B$. breve alone group), moderate in the LAC-RAF group (1.4\%) and high in the LAC-RAF-GOS group (6.0\%) (Fig. 2(B)). The amount of acetate in the caecum was significantly higher in the LAC-RAF-GOS group than in other groups $(P<0.05)$ (Fig. 2(C)). We also analysed the microbiota of these neonatal mice using real-time PCR with primers specific to the $16 \mathrm{~S}$ rRNA gene of each bacterial group. Compared with the vehicle group, the cell numbers of Enterobacteriaceae were higher in the $B$. breve alone, LAC and LAC-RAF groups, but remained unchanged in the LAC-RAF-GOS group (Fig. 2(D)). On the other hand, the cell numbers of Bacteroidetes were greater in the $B$. breve alone and LAC-RAF-GOS groups $(P<0.05)$ (Fig. 2(D)). Firmicutes were slightly greater in the $B$. breve alone group and were unchanged in the co-administered groups $(P<0 \cdot 05)$ (Fig. 2(D)). The cell numbers of Lactobacillus were comparable in all groups (Fig. 2(D)).

In the in vivo experiment, serum acetate concentrations were increased in the LAC-RAF-GOS group (Fig. 3(A)). We investigated mRNA levels of metabolism- and immune response-related genes in the liver and large intestine by quantitative real-time PCR. We found that the mRNA levels of gluconeogenesis- and fatty acid $\beta$-oxidation-related genes were unchanged in the liver of the LAC-RAF-GOS group, except for a slight increase in expression of the $\beta$-oxidation enzyme gene, Acadm, relative to other groups $(P<0.05)$ (Fig. 3(B)). On the other hand, mRNA levels of certain markers of helper T-cell (Th) subsets, such as Gata3 (marker of Th2) and Foxp3 (marker of regulatory T cell), but not T-bet (marker of Th1) or Rorc (marker of Th17), were increased in the large intestine of the LAC-RAF-GOS group relative to all other groups $(P<0.05)$ (Fig. 3(C)). In addition, levels of the innate immune sensor gene Tlr2, but not Tlr4, tended to be decreased and levels of the hormone gene $G c g$, which produces glucagon-like peptide (GLP)-1 and GLP-2 in the intestine, but not Pyy, were increased in the LAC-RAF-GOS group relative to all other groups $(P<0 \cdot 05)$ (Fig. 3(C)). 
(A)

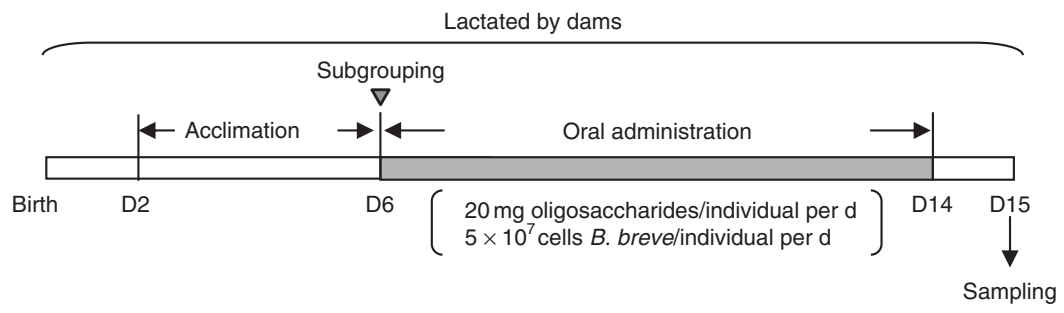

(B)

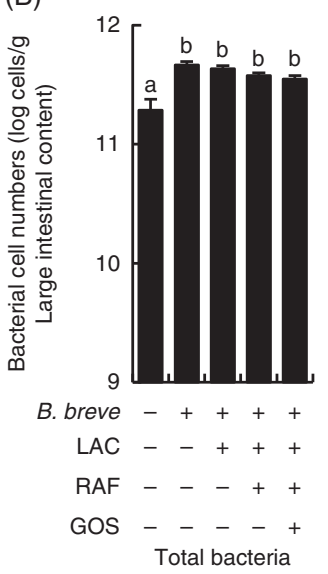

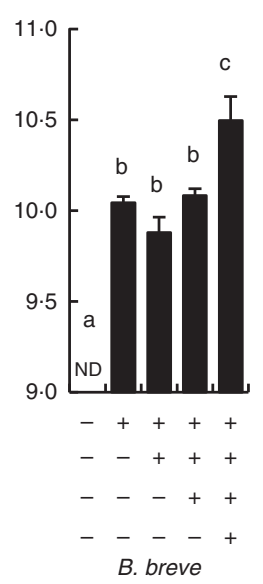

(C)
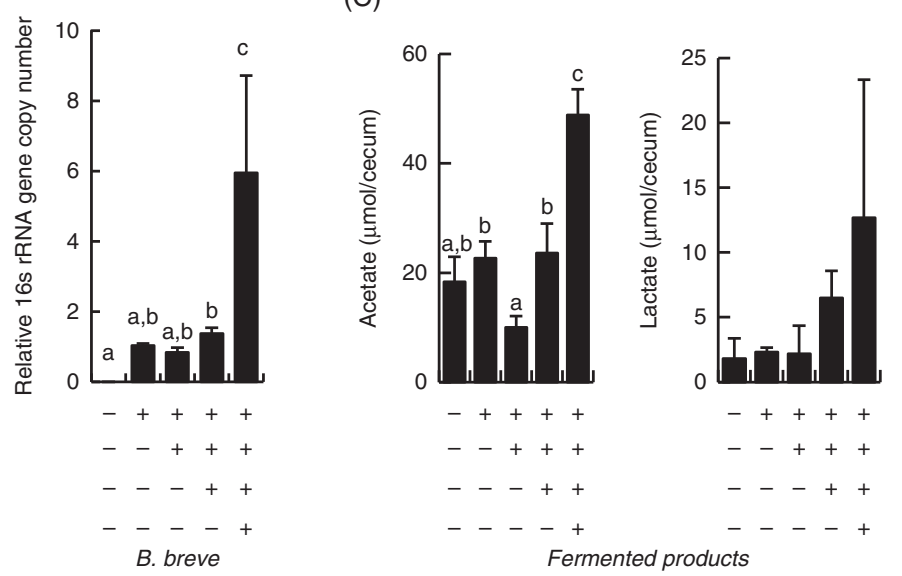

(D)
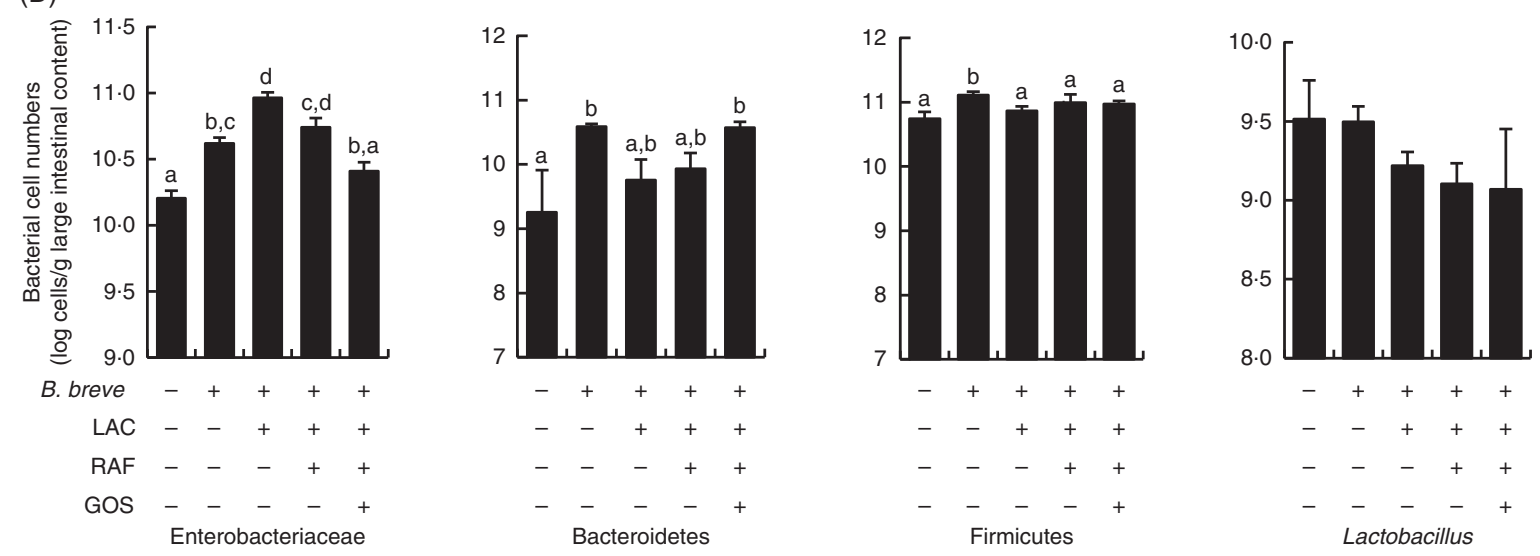

Fig. 2. Combinational effects of oligosaccharides on the bifidobacterial growth and gut microbiota in neonatal mice. (A) The protocol of the oral administration with oligosaccharides to neonatal mice. (B) 16S rRNA gene copy numbers of total bacteria (left panel), cell numbers of Bifidobacterium breve (middle panel) and 16S rRNA gene copy numbers of $B$. breve relative to those of total bacteria (right panel) in the large intestinal content of neonatal mice. Data were expressed as per gram wet weight of the large intestinal contents. (C) The amounts of lactate and acetate in the caecum of neonatal mice. (D) Cell numbers of Enterobacteriaceae, Bacteroidetes, Firmicutes and Lactobacillus in the large intestinal content of neonatal mice. Values are means ( $n$ 7-8), with their standard errors. ${ }^{\text {a,b,c,d }}$ Mean values with unlike letters were significantly different $(P<0.05$, Tukey-Kramer HSD test). ND, not detected; LAC, lactulose; RAF, raffinose; GOS, galacto-oligosaccharide.

\section{Discussion}

It is important to provide formula-fed infants with a bifidobacteria-enriched gut microbiota similar to that of breastfed infants. Various types of non-digestive oligosaccharides are used in infant formula worldwide to improve the gut microbiota of formula-fed infants, but a comparative validation of these oligosaccharides remains insufficient. In this study, we used an in vitro mixed culture model of artificial infant microbiota with seven bacterial species as a simple method to assess the bifidogenic effects of oligosaccharides alone and in combination. We found that the combination of LAC, RAF and GOS exhibited synergistic effects on the in vitro growth of $B$. breve and B. longum compared with LAC alone and LAC-RAF and LAC-GOS combinations. This synergistic bifidogenic effect was confirmed in the in vivo experiment using neonatal mice. Our results suggest that the LACRAF-GOS combination may be useful in infant formula to promote healthy gut microbiota in formula-fed infants.

Our in vitro mixed culture model of artificial infant microbiota well reflected the in vivo bifidogenic effect of oligosaccharides. This in vitro model may be useful to screen 
(A)

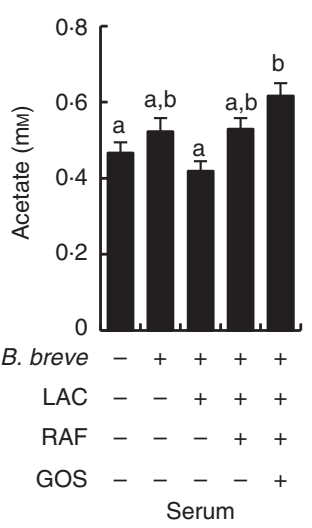

(B)

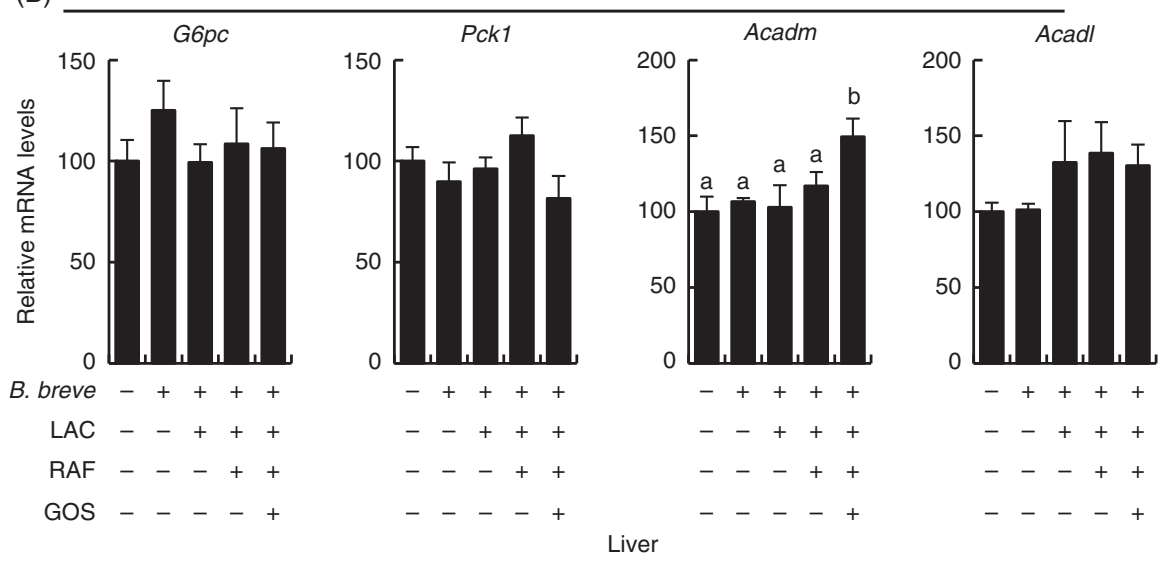

(C)
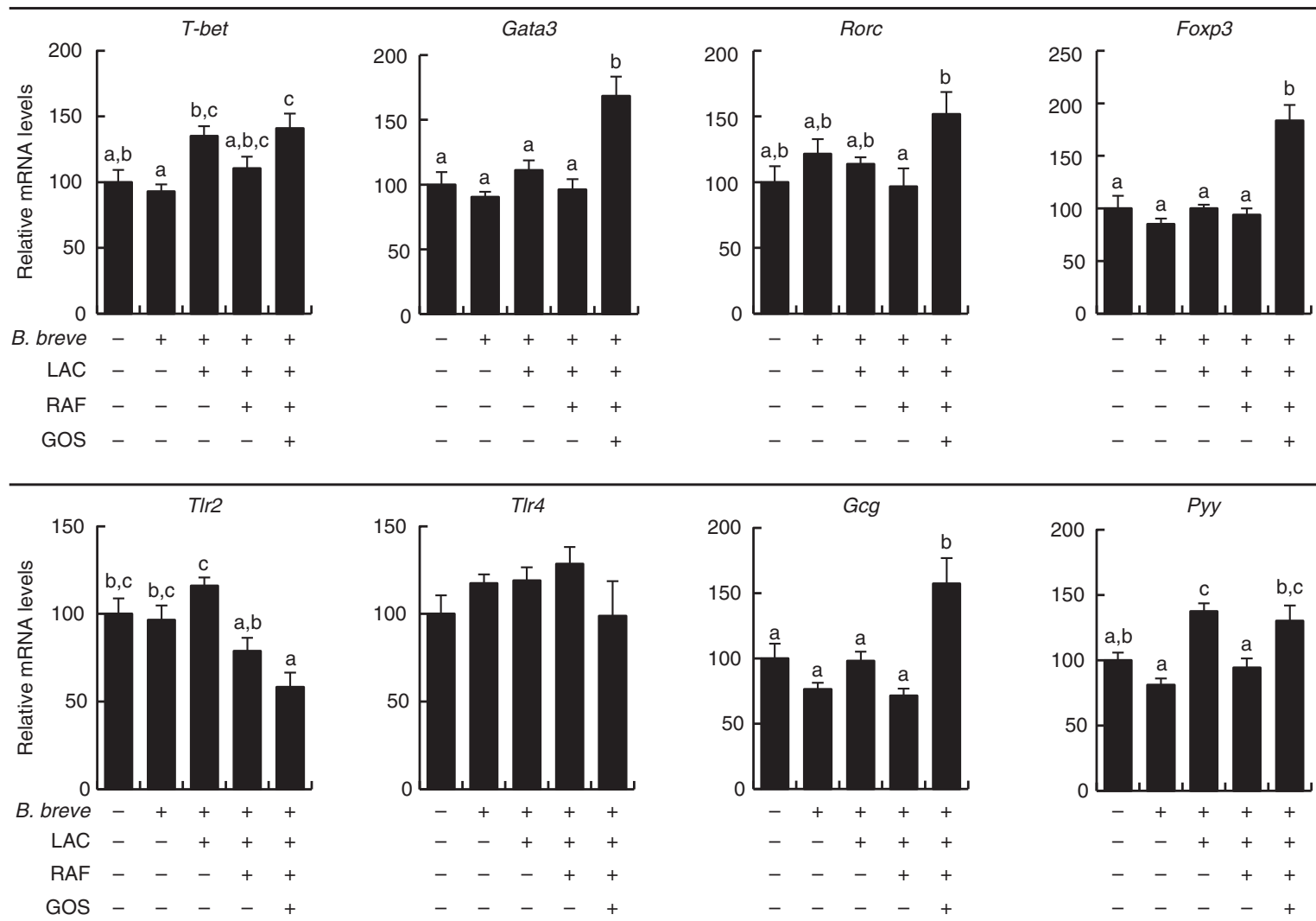

Fig. 3. Combinational effects of oligosaccharides on the serum acetate and gene expression in neonatal mice. (A) Serum acetate concentration. (B) Expression of genes related to gluconeogenesis (G6pc and Pck1) and fatty acid $\beta$-oxidation (Acadm and Acadl) in the liver. (C) Gene expression of helper T cell (Th) subset markers (T-bet, Gata3, Rorc and Foxp3), sensors of microbial components (T/r2 and T/r4) and gut-derived hormones (Gcg and Pyy) in the large intestine. Values are means ( $n$ 7-8), with their standard errors. ${ }^{\text {a,b,c }}$ Mean values with unlike letters were significantly different $(P<0.05$, Tukey-Kramer HSD test). LAC, lactulose; RAF, raffinose; GOS, galacto-oligosaccharide; G6pc, glucose-6-phosphatase, catalytic; Pck1, phosphoenolpyruvate carboxykinase 1, cytosolic; Acadm, acyl-coenzyme A dehydrogenase, medium chain; Acadl, acyl-coenzyme A dehydrogenase, long-chain; T-bet, T-box 21; Gata3, GATA binding protein 3; Rorc, RAR-related orphan receptor gamma; Foxp3, forkhead box P3; TIr2, Toll-like receptor 2; TIr4, Toll-like receptor 4; Gcg, glucagon; Pyy, peptide YY.

better oligosaccharides for infant. On the other hand, the in vitro model had some experimental limitations. For example, the in vitro model is unable to reproduce the indirect effects of oligosaccharides brought about by changes within the gut microbiota and metabolites, as well as by host-microbe interactions. In fact, cell numbers of Enterobacteriaceae (represented by E. coli in our culture model) were slightly increased in the LAC-RAF-GOS group relative to the LAC and LAC-RAF groups in the in vitro model, but were lower in the neonatal mice administered with LAC-RAF-GOS than in the mice administered with LAC and LAC-RAF. Therefore, our in vitro model is valid as a simple method to evaluate the direct bifidogenic effects of oligosaccharides and can serve as an alternative to faecal culture experiments for this purpose, but it 
may not be suitable for predicting overall changes in the infant gut microbiota caused by oligosaccharides.

We found that RAF and GOS exhibited different effects on the growth of bifidobacteria in combination with LAC in our in vitro model. LAC-RAF enhanced the growth of B. longum, whereas LAC-GOS enhanced the growth of $B$. breve, acetate production and $\mathrm{pH}$ reduction compared with LAC alone. This is consistent with the results of a previous report that $B$. breve highly utilised GOS compared with $B$. longum ${ }^{(34)}$. This is also consistent with a recent study showing that $B$. breve was effectively increased in the gut microbiota of infants fed formula containing GOS as the only prebiotic ${ }^{(35)}$. Interestingly, the features of LAC-RAF and LAC-GOS combinations remained or were emphasised in the LAC-RAF-GOS combination, which was high in both $B$. breve and $B$. longum cell numbers, high in acetate concentration and rapidly decreased in $\mathrm{pH}$. This was true even though the total oligosaccharide concentration was equal in all media (i.e. the initial concentration of each oligosaccharide was relatively low in the medium with three oligosaccharides compared with the medium with two oligosaccharides). The underlying mechanisms of this synergistic effect remain unknown, but secondary metabolites and inter-species metabolite transfers, as well as increased choices of carbohydrate sources, may be involved. Metabolomic and metatranscriptomic approaches could help understand the overall metabolic process of LACRAF-GOS in the in vitro model. Previous in vitro and animal studies reported that structurally different oligosaccharides exhibited different prebiotic effects and fermentation properties ${ }^{(19,36)}$. Over 200 structurally different oligosaccharides have been identified in human milk ${ }^{(20)}$. Taken together, these reports and our results suggest that an increased structural diversity of oligosaccharide combinations could enhance the bifidogenic effects of infant formula. The LAC-RAF-GOS combination also increased the cell numbers of Bacteroidetes in our in vivo experiment. Recent studies on the composition of the infant gut microbiota using high-throughput $16 \mathrm{~S}$ rRNA gene sequencing suggest that breastfed infants have more Bacteroidetes than formula-fed infants ${ }^{(37,38)}$. Although results from our in vivo experiment must be carefully interpreted because of the differences in gut microbiota between neonatal mice and human infant (dominated by Enterobacteriaceae and containing few bifidobacteria in neonatal mice $)^{(39,40)}$, formula with a LACRAF-GOS combination could contribute to the development of gut microbiota in formula-fed infants similar to that in breastfed infants by increasing bifidobacteria and Bacteroidetes.

In our in vivo experiment, we also observed that oral administration of the three-oligosaccharide combination increased acetate concentrations in the caecum and serum of neonatal mice. Expression of the fatty acid $\beta$-oxidation gene, Acadm, was increased in the liver, and expression of some Th subset markers, including Foxp3 and proglucagon gene $G c g$, were increased in the large intestine, suggesting an altered metabolism and immune response in the neonatal mice administered with the three-oligosaccharide combination. With regard to the physiological significance of these observations, there are reports indicating that SCFA, such as acetate, produced by the gut microbiota can prevent the development of host metabolic disorders by several mechanisms, such as by elevating hepatic fatty acid oxidation and intestinal Gcg expression/GLP-1 secretion ${ }^{(41-43)}$. In addition, recent studies indicate that gut microbiota-derived SCFA can suppress inflammatory and allergic responses by inducing the differentiation of colonic regulatory $\mathrm{T}$ cells ${ }^{(44)}$ or by enhancing the cell numbers and function of lung regulatory $\mathrm{T}$ cells ${ }^{(45)}$ via epigenetic modification and induction of the Foxp3 gene ${ }^{(44,45)}$. Some reports suggest that formula feeding increases the risk of obesity in childhood and later in life ${ }^{(46,47)}$ and also increases risks of infection ${ }^{(10-13)}$ and allergies ${ }^{(14)}$ relative to exclusive breastfeeding. Considering the apparent differences between the gut microbiota of breastfed and formula-fed infants, correcting the gut microbiota with the three-oligosaccharide combination could improve host metabolism and immune responses of formula-fed infants. Interestingly, the disruption of the gut microbiota at the neonatal stage results in later metabolic diseases $^{(48)}$, and early-life exposure to acetate prevents later development of allergic airways diseases ${ }^{(45)}$ in animal models. Further studies using pathological models are needed to clarify the effects of intervention in the infant gut microbiota with prebiotic oligosaccharides on long-lasting health.

In conclusion, this study reveals a synergistic bifidogenic effect of the three-oligosaccharide combination, both in vitro and in vivo, providing valuable information for the development of infant formula, as well as for management of the gut microbiota and health of formula-fed infants.

\section{Acknowledgements}

The authors thank Dr Xiao, Dr Odamaki, Dr Sato and Sugahara of the Morinaga Milk Industry Co. Ltd for technical support in the in vitro fermentation experiment and in the gut microbiota analysis, and thank Hatano and Tsutsumi of the Morinaga Milk Industry Co. Ltd for technical support in the analysis of lactulose.

T. E., H. I., K. N. and Y. T. designed the study. T. E., H. I. and M. T. performed the experiments and T. E. analysed the data. T. E., H. I., M. T., H. I., K. N. and Y. T. wrote the manuscript. This work was funded by Morinaga Milk Industry Co., Ltd. All the authors read and approved the final manuscript. Morinaga Milk Industry Co. Ltd had no role in the design, analysis or writing of this article.

All authors are employees of, and obtain salaries from, Morinaga Milk Industry Co. Ltd Morinaga Milk Industry Co. Ltd provided the $B$. breve $\mathrm{M}-16 \mathrm{~V}$ and lactulose used in this study.

\section{Supplementary material}

For supplementary material/s referred to in this article, please visit http://dx.doi.org/doi:10.1017/S0007114516001987

\section{References}

1. Koropatkin NM, Cameron EA \& Martens EC (2012) How glycan metabolism shapes the human gut microbiota. Nat Rev Microbiol 10, 323-335.

2. Harmsen HJ, Wildeboer-Veloo AC, Raangs GC, et al. (2000) Analysis of intestinal flora development in breast-fed and 
formula-fed infants by using molecular identification and detection methods. J Pediatr Gastroenterol Nutr $\mathbf{3 0}$, 61-67.

3. Fukuda S, Toh H, Hase K, et al. (2011) Bifidobacteria can protect from enteropathogenic infection through production of acetate. Nature $\mathbf{4 6 9}, 543-547$.

4. Yamashiro Y \& Nagata S (2010) Beneficial microbes for premature infants, and children with malignancy undergoing chemotherapy. Benef Microbes 1, 357-365.

5. Taipale T, Pienihakkinen K, Isolauri E, et al. (2011) Bifidobacterium animalis subsp. lactis BB-12 in reducing the risk of infections in infancy. Br J Nutr 105, 409-416.

6. Kalliomaki M, Kirjavainen P, Eerola E, et al. (2001) Distinct patterns of neonatal gut microflora in infants in whom atopy was and was not developing. I Allergy Clin Immunol 107, $129-134$.

7. Enomoto T, Sowa M, Nishimori K, et al. (2014) Effects of bifidobacterial supplementation to pregnant women and infants in the prevention of allergy development in infants and on fecal microbiota. Allergol Int 63, 575-585.

8. Mohan R, Koebnick C, Schildt J, et al. (2008) Effects of Bifidobacterium lactis Bb12 supplementation on body weight, fecal $\mathrm{pH}$, acetate, lactate, calprotectin, and IgA in preterm infants. Pediatr Res 64, 418-422.

9. de Kivit S, Saeland E, Kraneveld AD, et al. (2012) Galectin-9 induced by dietary synbiotics is involved in suppression of allergic symptoms in mice and humans. Allergy 67, 343-352.

10. Hylander MA, Strobino DM \& Dhanireddy R (1998) Human milk feedings and infection among very low birth weight infants. Pediatrics 102, E38.

11. Oddy WH, Sly PD, de Klerk NH, et al. (2003) Breast feeding and respiratory morbidity in infancy: a birth cohort study. Arch Dis Child 88, 224-228.

12. Tarrant M, Kwok MK, Lam TH, et al. (2010) Breast-feeding and childhood hospitalizations for infections. Epidemiology 21, 847-854.

13. Sinha A, Madden J, Ross-Degnan D, et al. (2003) Reduced risk of neonatal respiratory infections among breastfed girls but not boys. Pediatrics 112, e303.

14. Saarinen UM \& Kajosaari M (1995) Breastfeeding as prophylaxis against atopic disease: prospective follow-up study until 17 years old. Lancet 346, 1065-1069.

15. Newburg DS \& Walker WA (2007) Protection of the neonate by the innate immune system of developing gut and of human milk. Pediatr Res 61, 2-8.

16. Lonnerdal B (2003) Nutritional and physiologic significance of human milk proteins. Am J Clin Nutr 77, 1537S-1543S.

17. Sela DA, Chapman J, Adeuya A, et al. (2008) The genome sequence of Bifidobacterium longum subsp. infantis reveals adaptations for milk utilization within the infant microbiome. Proc Natl Acad Sci U S A 105, 18964-18969.

18. German JB, Freeman SL, Lebrilla CB, et al. (2008) Human milk oligosaccharides: evolution, structures and bioselectivity as substrates for intestinal bacteria. Nestle Nutr Workshop Ser Pediatr Program 62, 205-218; discussion 218-222.

19. Xiao JZ, Takahashi S, Nishimoto M, et al. (2010) Distribution of in vitro fermentation ability of lacto-N-biose I, a major building block of human milk oligosaccharides, in bifidobacterial strains. Appl Environ Microbiol 76, 54-59.

20. Zivkovic AM, German JB, Lebrilla CB, et al. (2011) Human milk glycobiome and its impact on the infant gastrointestinal microbiota. Proc Natl Acad Sci U S A 108, Suppl. 1, 4653-4658.

21. Garrido D, Barile D \& Mills DA (2012) A molecular basis for bifidobacterial enrichment in the infant gastrointestinal tract. Adv Nutr 3, 415S-421S
22. Watson D, O'Connell Motherway M, Schoterman MH, et al. (2013) Selective carbohydrate utilization by lactobacilli and bifidobacteria. J Appl Microbiol 114, 1132-1146.

23. Kiyosawa I, Takase M, Yamauchi K, et al. (1986) Lactulose and intestinal microflora in infant nutrition. Bifidobacteria Microflora 5, 27-35.

24. Elkington SG (1970) Lactulose. Gut 11, 1043-1048.

25. Hattori K, Sasai M, Yamamoto A, et al. (2000) Intestinal flora of infants with cow milk hypersensitivity fed on casein-hydrolyzed formula supplemented raffinose. Arerugi 49, 1146-1155.

26. Williams T, Choe Y, Price P, et al. (2014) Tolerance of formulas containing prebiotics in healthy, term infants. J Pediatr Gastroenterol Nutr 59, 653-658.

27. Veereman G (2007) Pediatric applications of inulin and oligofructose. J Nutr 137, 2585S-2589S.

28. Xia Q, Williams T, Hustead D, et al. (2012) Quantitative analysis of intestinal bacterial populations from term infants fed formula supplemented with fructo-oligosaccharides. J Pediatr Gastroenterol Nutr 55, 314-320.

29. Satoh $\mathrm{T}$, Odamaki $\mathrm{T}$, Namura $\mathrm{M}$, et al. (2013) In vitro comparative evaluation of the impact of lacto-N-biose I, a major building block of human milk oligosaccharides, on the fecal microbiota of infants. Anaerobe 19, 50-57.

30. Rossi M, Corradini C, Amaretti A, et al. (2005) Fermentation of fructooligosaccharides and inulin by bifidobacteria: a comparative study of pure and fecal cultures. Appl Environ Microbiol 71, 6150-6158.

31. Martin FP, Wang Y, Sprenger N, et al. (2008) Probiotic modulation of symbiotic gut microbial-host metabolic interactions in a humanized microbiome mouse model. Mol Syst Biol 4, 157.

32. Abe F, Muto M, Yaeshima T, et al. (2010) Safety evaluation of probiotic bifidobacteria by analysis of mucin degradation activity and translocation ability. Anaerobe 16, 131-136.

33. Hardy MR, Townsend RR \& Lee YC (1988) Monosaccharide analysis of glycoconjugates by anion exchange chromatography with pulsed amperometric detection. Anal Biochem 170, 54-62.

34. Barboza M, Sela DA, Pirim C, et al. (2009) Glycoprofiling bifidobacterial consumption of galacto-oligosaccharides by mass spectrometry reveals strain-specific, preferential consumption of glycans. Appl Environ Microbiol 75, 7319-7325.

35. Sierra C, Bernal MJ, Blasco J, et al. (2015) Prebiotic effect during the first year of life in healthy infants fed formula containing GOS as the only prebiotic: a multicentre, randomised, double-blind and placebo-controlled trial. Eur J Nutr 54, 89-99.

36. Van Craeyveld V, Swennen K, Dornez E, et al. (2008) Structurally different wheat-derived arabinoxylooligosaccharides have different prebiotic and fermentation properties in rats. J Nutr 138, 2348-2355.

37. Fan W, Huo G, Li X, et al. (2014) Impact of diet in shaping gut microbiota revealed by a comparative study in infants during the six months of life. J Microbiol Biotechnol 24, 133-143.

38. Donovan SM, Wang M, Li M, et al. (2012) Host-microbe interactions in the neonatal intestine: role of human milk oligosaccharides. Adv Nutr 3, 450S-455S.

39. Mirpuri J, Raetz M, Sturge CR, et al. (2014) Proteobacteriaspecific IgA regulates maturation of the intestinal microbiota. Gut Microbes 5, 28-39.

40. Gomez-Gallego C, Collado MC, Perez G, et al. (2014) Resembling breast milk: influence of polyaminesupplemented formula on neonatal BALB/cOlaHsd mouse microbiota. Br J Nutr 111, 1050-1058.

41. Kondo T, Kishi M, Fushimi T, et al. (2009) Acetic acid upregulates the expression of genes for fatty acid oxidation enzymes in liver to suppress body fat accumulation. J Agric Food Chem 57, 5982-5986. 
42. Yadav H, Lee JH, Lloyd J, et al. (2013) Beneficial metabolic effects of a probiotic via butyrate-induced GLP-1 hormone secretion. J Biol Chem 288, 25088-25097.

43. den Besten G, van Eunen K, Groen AK, et al. (2013) The role of short-chain fatty acids in the interplay between diet, gut microbiota, and host energy metabolism. J Lipid Res 54, 2325-2340.

44. Furusawa Y, Obata Y, Fukuda S, et al. (2013) Commensal microbe-derived butyrate induces the differentiation of colonic regulatory T cells. Nature 504, 446-450.

45. Thorburn AN, McKenzie CI, Shen S, et al. (2015) Evidence that asthma is a developmental origin disease influenced by maternal diet and bacterial metabolites. Nat Commun 6, 7320 .

46. Yamakawa M, Yorifuji T, Inoue S, et al. (2013) Breastfeeding and obesity among schoolchildren: a nationwide longitudinal survey in Japan. JAMA Pediatr 167, 919-925.

47. Oddy WH, Mori TA, Huang RC, et al. (2014) Early infant feeding and adiposity risk: from infancy to adulthood. Ann Nutr Metab 64, 262-270.

48. Cox LM, Yamanishi S, Sohn J, et al. (2014) Altering the intestinal microbiota during a critical developmental window has lasting metabolic consequences. Cell 158, 705-721. 